\title{
IMMUNOHISTOCHEMICAL EXPRESSION OF MUCIN 1AND MUCIN 2 IN INFLAMMATORY AND NEOPLASTIC LESIONS OF THE COLON
}

\author{
Khaled E.Soliman ${ }^{(1)}$, Malak A.Zoheir ${ }^{(2)}$, Hoda A.Helmy ${ }^{(2)}$, Aisha A.Essam Eldin ${ }^{(2)}$ \\ ${ }^{(1)}$ Surgery Department, ${ }^{(2)}$ Pathology Department, Medical Research Institute, Alexandria University, Egypt.
}

\section{ABSTRACT:}

Background: The mucus layer coating the gastrointestinal tract is the front line of innate host defense. MUC2 is the major secretory mucin synthesized and secreted by goblet cells, whereas goblet and absorptive cells express membrane-bound mucins such as MUC1 in the apical membrane. MUC1 plays a role in tumorigenesis through changes in intracellular signaling, adhesion and migration and by increasing resistance to apoptosis. MUC2 gene product either functions as a tumor suppressor or contributes to the function of a tumor suppressor.

Aim: To investigate the expression profile of MUC1 and MUC2 in normal, inflammed and neoplastic lesions of the colon and also to analyze the immunohistochemical expression of MUC1 and MUC2 and their relationship to the site, histological differentiation and stage of colorectal carcinoma.

Patients and Methods: Thirty patients with colorectal carcinomas underwent surgical resections, 15 patients underwent endoscopically resected colorectal adenoma, 15 patients subjected to endoscopic biopsy specimens of ulcerative colitis and a 10 control cases of colorectal specimens taken from the free resection margins of colectomy specimens were included in this study.All tissue specimens were subjected to MUC1 and MUC2 immunohistochemical staining using monoclonal antibodies.

Results:The positive expression rates of MUC1 and MUC2 in colorectal carcinoma were $76.7 \%$ and $60 \%$ respectively.MUC1 immunoreactivity was detected in $33.3 \%$ of colorectal adenomas whereas MUC2 expression was observed in $93.3 \%$ of cases.MUC2 expression was noted in all cases of ulcerative colitis(100\%), while MUC1 was expressed in $40 \%$ of cases. The expression rates of MUC1 and MUC2 in control cases were zero and $100 \%$ respectively.MUC1 expression was significantly higher in carcinomas as compared with UC and adenomas, while MUC2 expression was significantly lower in carcinomas as compared with UC and adenomas.MUC1 expression was significantly higher in colorectal adenocarcinomas, whereas MUC2 was significantly expressed in mucinous carcinomas $(\mathrm{P} \leq 0.05)$. No significant correlation was found between expression of MUC1 or MUC2 and histological grade ( $\mathrm{P}>0.05$ ).High significant association was found between advanced tumor stage and MUC1 expression $(\mathrm{P} \leq 0.05)$, while MUC2 expression was significantly correlated with lower tumor stages.No significant correlation was found between expression of MUC1or MUC2 and tumor location ( $\mathrm{P}>0.05)$

Conclusion:We conclude that, Up-regulation of MUC1 and downregulation of MUC2 expression could be involved in carcinogenesis and progression in colorectal tumors and reflect the prognosis to a certain extent. However, further studies of mucin changes in cancer and inflammation are warranted not only as diagnostic and prognostic markers but also as therapeutic targets.

\section{INTRODUCTION}

Golor olorectal cancer is the second most common cause of cancer related death in the developed world, in consequence advances in our understanding and treatment of colorectal cancers can potentially have a huge impact on cancer morbidity and mortality. ${ }^{(1)}$ At present TNM stage, tumor type and resection margin status are the most widely used parameters in planning adjuvant treatment. Tumor grade of differentiation, vascular invasion and more recently perineural invasion and tumor border configuration have also been used to assist the clinician in predicting colorectal tumor behavior and hence subsequent patient management. Refining prognostic markers allow treatment to be more accurately tailored to individual patients, as well as suggesting potential mechanisms through which tumor progression occurs which in turn could provide targets for novel therapies. ${ }^{(2)}$

There are many cases of cancer in the gastrointestinal tract which develop from a precancerous lesions. This brings a window of opportunity to look at the steps of development of cancer and also for patients to be scheduled for surveillance procedures and to get their cancer diagnosed earlier and subsequently having earlier eradication. This fact is clear indeed in colorectal cancer which is considered as one of the most preventable cancers because it might develop from various pathological conditions like inflammatory bowel

Key Words: MUC1-MUC2- Inflammatory and Colorectal tumors- Immunostaining 
diseases especially ulcerative colitis and adenomatous polyps. The transformation rate of adenomatous polyps into carcinoma is about $0.25 \%$ per year ${ }^{(3)}$

Mucins are large glycoproteins composed of $75 \%$ carbohydrate and $25 \%$ amino acids linked via oglycosidic bonds. The human mucin (MUC) family consists of 21 mucin genes of which 15 are expressed in different regions of the gastrointestinal tract. $\left.{ }^{(4,5}\right)$ Mucin 1 (MUC1) is an epithelial cell glycoprotein that is expressed by almost all glandular epithelial surfaces of respiratory, salivary gland, mammary gland and pancreatic epithelium. ${ }^{(6)}$ Its expression is dramatically increased in breast, ovarian, lung, prostate and pancreatic cancers. ${ }^{(7)}$ MUC1 plays a role in tumorigenesis through changes in intracellular signaling, adhesion and migration, and by increasing resistance to apoptosis. ${ }^{(8)}$ Mucin2 (MUC2) is the predominant mucin produced by intestinal goblet cells which are most frequent in healthy colon. ${ }^{(9)}$ MUC2 gene product either functions as a tumor suppressor or contributes to the function of a tumor suppressor. ${ }^{(10)}$ MUC2 is expressed by adenomas and mucinous carcinomas. Down-regulation of MUC2 is seen in non mucinous adenocarcinomas arising within adenomas. ${ }^{(11)}$

The aim of this study is to investigate the expression profile of MUC1 and MUC2 in normal, inflammed and neoplastic lesions of the colon and also to analyze the immunohistochemical expression pattern of MUC1and MUC2 and their relationship to the site, histological grade and stage of colorectal carcinoma.

\section{PATIENTS AND METHODS:}

The current study was carried out on a total of 30 patients with colorectal carcinoma undergoing tumor resection at the Department of Surgery, 15 patients undergoing endoscopically resected colorectal adenoma, 15 patients subjected to colonoscopic biopsies of ulcerative colitis at the Endoscopy unit, and a 10 control cases of colorectal specimens taken from the free resection margin of the colectomy specimens, Medical Research Institute, Alexandria University, Egypt, in the period between January 2014 and July 2015.

Patients with colorectal carcinoma included 4 patients with cancer caecum, 8 patients with cancer ascending colon, 7 patients with cancer descending colon and 11 patients with cancer rectosigmoid. Before surgery, all patients were subjected to complete clinical examination, routine laboratory investigations, radiological investigations (chest x-ray, abdominal US and CT scan abdomen and pelvis) and colonoscopic biopsy for pathological examination. Surgical interventions included right hemicolectomy for patients with cancer caecum and ascending colon, left hemicolectomy for patients with cancer descending colon and anterior resection for patients with cancer rectosigmoid. After surgery, the surgical specimens were sent to the Pathology 9.5
At the Pathology Department, the specimens received were allocated into 4 groups:

Group I: Thirty specimens of surgically resected colorectal cancer.

Group II: Fifteen specimens of endoscopically resected colorectal adenoma.

GroupIII: Fifteen endoscopic biopsy specimens of ulcerative colitis. Diagnosis of ulcerative colitis was based on clinical symptoms, colonoscopy and histological findings.

Group IV: Ten control colorectal specimens collected from the free resection margins of colectomy specimens.

\section{Pathological assessment:}

$5 \mu \mathrm{m}$ thick representative paraffin-embedded tissue sections from each case were subjected to Hematoxylin and Eosin $(\mathrm{H} \& \mathrm{E})$ staining for reviewing the diagnosis of the lesion and histological grading of tumors according to WHO classification. ${ }^{(12)}$ Tumor and lymph nodes sections were staged according to the TNM staging from AJCC $2010 .^{(13)}$

\section{MUC1 and MUC2 immunohistochemical staining using monoclonal antibodies:}

$5 \mu \mathrm{m}$ sections were mounted on coated slides, deparaffinised with xylene, gradually rehydrated in descending grades of alcohol and then rinsed in distilled water. Activity of endogenous peroxidases was blocked by $30 \mathrm{~min}$. incubation in $3 \% \mathrm{H} 2 \mathrm{O} 2$ to prevent non specific binding of antibody. Antigen retrieval was performed by heating in a microwave oven at $800 \mathrm{~W}$ in citrate buffer ( PH 6.0) for 15 minutes in thermo-resistant container. Buffer was added periodically to the container to prevent drying during incubation process. Slides were cooled in buffer for 20 minutes, then washed twice in phosphate buffered saline (PBS). Immunohistochemical reaction was performed using Monoclonal mouse antibody against MUC1 (SPM 493, 1:50, Spring Bioscience, USA), and Monoclonal mouse antibody against MUC2 (SPM 512, 1:200, Spring Bioscience, USA). ${ }^{(14}$ ) Sections were incubated with antibodies over night at room temperature. Subsequently, incubation was performed with Streptavidin-biotin peroxidase complex. ${ }^{(15)}$ All sections were counterstained with Meyers Hematoxylin and examined using the light microscope. External positive controls consisted of invasive breast carcinoma known to express MUC1 and small intestine for MUC2 monoclonal antibodies. Negative controls staining were obtained by replacing the primary antibody with PBS.

\section{Interpretation of immunohistochemical results:}

MUC1 and MUC2 staining were noted as brown stain in the cytoplasm or both the cytoplasm and membrane of colorectal epithelial cells. The level of staining for each 
mucin was calculated by determining the percentage of immunostained cells per high power field, and reported as 0 (no staining), $1+($ up to $30 \%), 2+(30-60 \%$ of cells stained $), 3+($ more than $60 \%$ of cells stained $)$. Clinicopathological data were compared according to presence (positivity) or absence (negativity) of immunehistochemical staining for each mucin. $\left({ }^{14)}\right.$

\section{STATISTICAL ANALYSIS}

Data were fed to the computer using IBM SPSS software package version 20. Qualitative data were analyzed using Chi-Square test. Fisher exact test and Monte Carlo tests were applied for Chi Square test. Values of $\mathrm{P} \leq 0.05$ were considered statistically significant.

\section{RESULTS}

The mean age of patients with colorectal carcinoma (Group I) was 50.1 \pm 15.09 years, range (23-78) years, 18 cases were males $(60 \%)$ and 12 were females (40\%) with male to female ratio $(3: 2)$. Twelve cases $(40 \%)$ were right sided colonic cancer ( caecum \&ascending colon) while 18 cases $(60 \%)$ were left sided colonic cancer (descending \&rectosigmoid colon). Regarding histological type, 21 cases $(70 \%)$ were adenocarcinomas with varying grades, while 9 cases $(30 \%)$ were mucinous carcinoma. Adenocarcinomas were graded into; 17 cases $(80 \%)$ were low grade (well \&moderately differentiated adenocarcinoma) and 4 cases $(20 \%)$ were high grade (poorly differentiated adenocarcinoma). TNM staging of colorectal carcinoma (CRC) cases were 5 cases $(16.7 \%)$ were stage I, 10 cases $(33.3 \%)$ were stage II and 15 cases $(50 \%)$ were stage III.

Regarding colorectal adenoma cases (Group II) , their mean age was $55.9 \pm 10.96$ years, range $(35-70)$ years. Eleven cases were males $(73.3 \%)$ while 4 cases were females $(26.7 \%)$. Histological examination of $\mathrm{H} \& \mathrm{E}$ stained sections confirmed that all 15 cases were tubulovillous adenomas. Ten cases $(66.7 \%)$ showed low grade dysplasia, while the remaining 5 cases $(33.3 \%)$ showed high grade dysplasia.

The mean age of patients with ulcerative colitis (Group III) was $36,5 \pm 3,98$ years, range (31-40) years. Nine cases were males $(60 \%)$ while the remaining 6 cases were females $(40 \%)$. Histological examination revealed mucosal ulceration with mild goblet cell depletion, cryptitis, diffuse inflammatory infiltrate, but no dysplasia was observed.

\section{Immunohistochemical staining results in the studied cases:}

In colorectal carcinoma cases, positive expression of MUC1was detected in 23 cases $(76.7 \%)$ with varying staining intensity ( 6 cases $+1,6$ cases $+2,11$ cases +3 ) while it was negatively expressed in 7 cases $(23.3 \%)$. On the other hand, MUC2 was positively expressed in 18 cases $(60 \%)$ with different staining pattern $(3$ cases $+1,8$ cases $+2,7$ cases +3 ) while negative expression for MUC2 was observed in 12 cases (40\%).

Regarding colorectal adenoma cases, immunehistochemical expression of MUC1 was noted in 5 cases $(33.3 \%)$ while the remaining 10 cases $(66.7 \%)$ showed no immunoreactivity for MUC1. The positive cases for MUC1 expression (5 cases) were adenoma with high grade dysplasia whereas, the negative cases for its expression (10 cases) were adenoma with low grade dysplasia. Also, MUC2 was expressed in 14 cases $(93.3 \%)$ of colorectal adenoma, but only one case $(6.7 \%)$ was negative for MUC2 expression.

As regards cases of ulcerative colitis (UC), MUC1 was expressed in 6 out of 15 cases $(40 \%)$ of UC, whereas 9 cases $(60 \%)$ were negative for MUC1 expression. On the other hand, all cases of UC (100\%) showed positive MUC2 expression.

Regarding control cases. all 10 normal colonic mucosal specimens were negative for MUC1 expression but all were positive for MUC2 expression.

The statistical analysis of the three studied groups compared together revealed a highly significant difference in MUC1 expression $(\mathrm{P} \leq 0.05)$. MUC1 was more frequently expressed in CRC than in UC and colorectal adenomas. (Table 1, Fig. 1)

The statistical analysis of the three studied groups compared together showed a significant difference in MUC2 expression $(\mathrm{P} \leq 0.05)$. MUC2 was more frequently expressed in UC and colorectal adenomas than in CRC. (Table 2, Fig. 1 )

\section{Relationship between MUC1 expression and clinicopathological parameters in CRC cases:}

Regarding tumor location, MUC1 was expressed in 15 out of 18 cases $(83.3 \%)$ of left sided colorectal carcinoma, whereas it was expressed in 8 out of 12 cases $(66.7 \%)$ of right sided colonic carcinoma. Statistical analysis revealed that there was no statistical significant association between MUC1 expression and tumor location $(\mathrm{P}>0.05)$. (Table 3)

As regards histologic type, MUC1 was expressed in 3 out of 9 cases $(33.3 \%)$ of mucinous carcinoma while it was negatively expressed in the remaining 6 cases $(66.7 \%)$. Out of 21 cases of colonic adenocarcinomas, 20 cases $(95.2 \%)$ showed MUC1 expression whereas, only one case $(4.8 \%)$ was negative for MUC1 expression.

Statistical analysis revealed that a statistically highly significant association was found between MUC1 expression and histologic type of CRC $(\mathrm{P} \leq 0.05)$. MUC1 was more frequently expressed in colonic adenocarcinomas than in mucinous carcinomas. (Table 3, Fig 1) 
Regarding histologic grade, MUC1 was expressed in 16 out of 17 cases $(94.1 \%)$ of low grade adenocarcinoma, while only one case $(5.9 \%)$ was negative for MUC1 expression. On the other hand, MUC1 was expressed in all 4 cases $(100 \%)$ of high grade adenocarcinoma.

Statistical analysis revealed that there was no statistical significant association between MUC1 expression and histologic grade $(\mathrm{P}>0.05)$. (Table 3$)$

As regards tumor stage, MUC1 expression was observed in 1 out of 5 cases $(20 \%), 8$ out of 10 cases $(80 \%)$ and 14 out of 15 cases $(93.3 \%)$ in stages I,II\&III respectively. (Table 3)

Statistical analysis revealed that there was statistically highly significant association between MUC1 expression and tumor stage in $\mathrm{CRC}(\mathrm{P} \leq 0.05)$. MUC1 was more frequently expressed in stage III than in stages II and I.

\section{Relationship between MUC2 expression and clinicopathological parameters in CRC cases:}

Regarding tumor site, MUC2 was expressed in 12 out of 18 cases $(66.7 \%)$ of left sided colorectal carcinomas, whereas, it was expressed in 6 out of 12 cases $(50 \%)$ of right sided colonic cancer.

Statistical analysis showed no statistical significant correlation between MUC2 expression and tumor site $(\mathrm{P}>0.05)$. (Table 4)
As regards histologic type, MUC2 was expressed in all 9 cases $(100 \%)$ of mucinous carcinoma. Out of 21 cases of colorectal adenocarcinomas, 9 cases $(42.9 \%)$ showed MUC2 expression, while the remaining 12 cases $(57.1 \%)$ were negative for MUC2 expression.

Statistical analysis showed that there was a statistically highly significant correlation between MUC2 expression and histologic type of CRC $(\mathrm{P} \leq 0.05)$. MUC2 was more frequently expressed in mucinous carcinomas than in colorectal adenocarcinomas. (Table 4, Fig. 1 )

Regarding histologic grade, MUC2 was expressed in 7 out of 17 cases $(41.2 \%)$ of low grade adenocarcinoma, whereas it was negatively expressed in 10 cases (58.8\%). On the other hand, MUC2 was expressed in 2 out of 4 cases $(50 \%)$ of high grade adenocarcinoma, while it was negatively expressed in the remaining 2 cases $(50 \%)$.

Statistical analysis showed no statistical significant correlation between MUC2 expression and histologic grade $(\mathrm{P}>0.05)$. (Table 4$)$

As regards tumor stage, MUC2 expression was detected in 4 of 5 cases $(80 \%), 9$ of 10 cases $(90 \%)$, and 5 of 15 cases $(33.3 \%)$ in stages I,II\&III of colorectal carcinomas respectively.

Statistical analysis showed that there was a statistically significant correlation between MUC2 expression and tumor stage in CRC $(\mathrm{P} \leq 0.05)$. MUC2 was more frequently expressed in stages I\&II than in stage III. (Table 4)

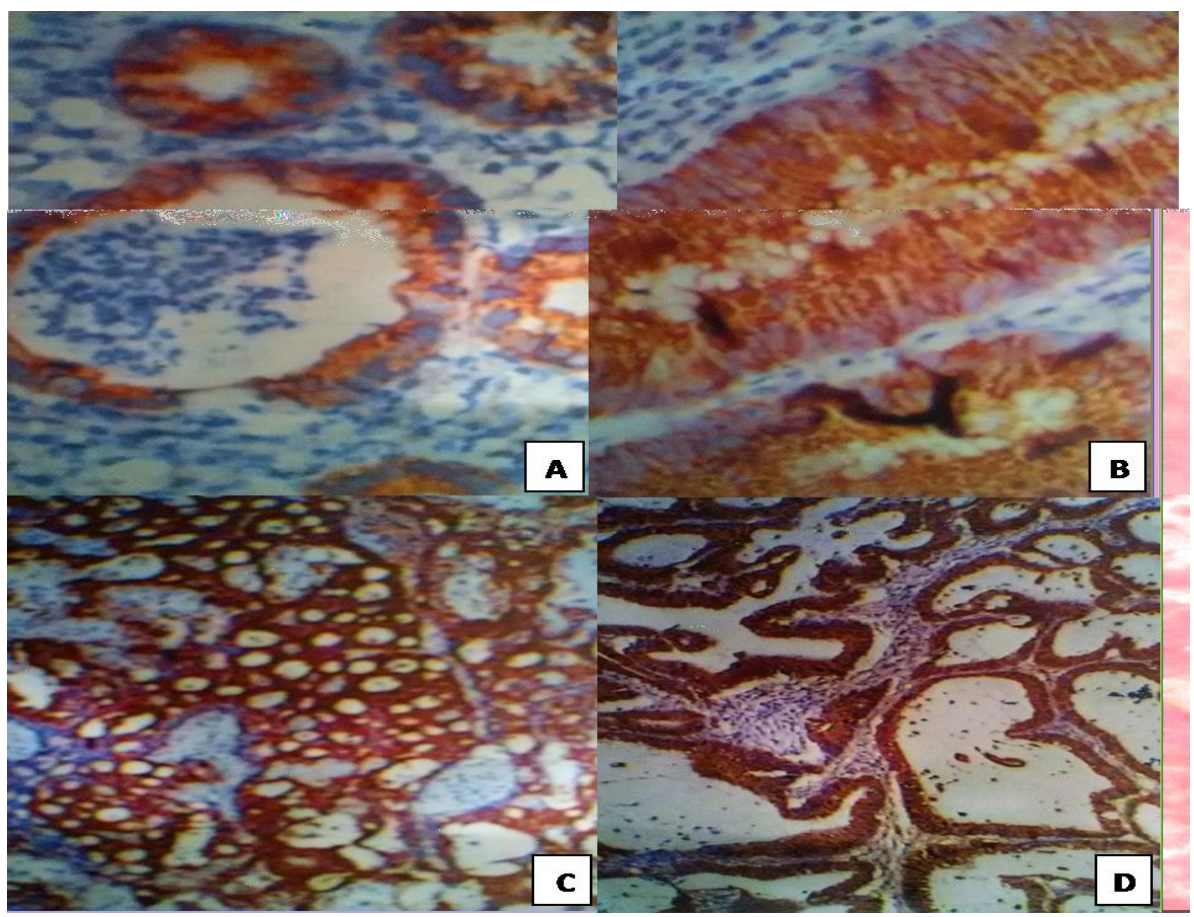

Figure 1:Showing the different immunohistochemical staining patterns of the studied cases regarding MUC1\& MUC2 expressions. A) Ulcerative colitis showing positive MUC1 expression score $(+2)$. B) Adenoma with low grade dysplasia showing positive MUC2 expression score $(+3)$. C) Low grade adenocarcinoma (moderately differentiated) showing positive MUC1 expression score (+2). D) Mucinous carcinoma showing positive MUC2 expression score $(+3)$. 
Table (1): Comparison between the studied groups according to MUC1 expression

\begin{tabular}{|c|c|c|c|c|c|c|c|c|}
\hline \multirow[b]{2}{*}{ MUC1 } & \multicolumn{2}{|l|}{$\mathbf{U C}$} & \multicolumn{2}{|c|}{ Adenoma } & \multicolumn{2}{|c|}{ Carcinoma } & \multicolumn{2}{|c|}{ Total } \\
\hline & No. & $\%$ & No. & $\%$ & No. & $\%$ & No. & $\%$ \\
\hline -ve & 9 & 60.0 & 10 & 66.7 & 7 & 23.3 & 26 & 43.3 \\
\hline$+v e$ & 6 & 40.0 & 5 & 33.3 & 23 & 76.7 & 34 & 56.7 \\
\hline Total & 15 & 100.0 & 15 & 100.0 & 30 & 100.0 & 60 & 100.0 \\
\hline$\chi^{2}$ & 9.91 & & & & & & & \\
\hline${ }^{\mathrm{MC}} \mathrm{p}$ & $.007 *$ & & & & & & & \\
\hline $\mathrm{P} 1$ & $0.015^{*}$ & & & & & & & \\
\hline $\mathrm{P} 2$ & $0.004 *$ & & & & & & & \\
\hline
\end{tabular}

Table (2): Comparison between the studied groups according to MUC2 expression.

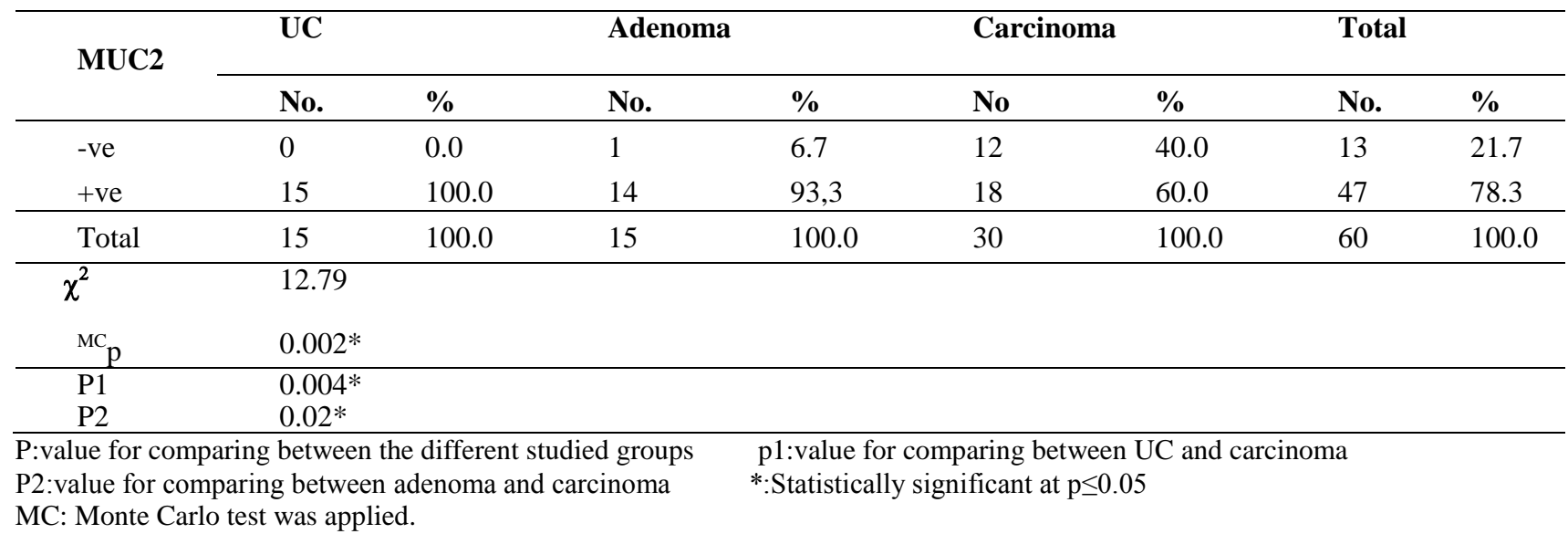

Table (3): Correlation between MUC1 expression and clinicopathological parameters in colorectal cancer cases

\begin{tabular}{|c|c|c|c|c|c|c|c|}
\hline \multirow{3}{*}{ Clinicopathological } & \multirow{3}{*}{ Variables } & \multicolumn{4}{|c|}{ MUC1 Immunoreactivity } & \multirow{3}{*}{$\chi^{2}$} & \multirow{3}{*}{$\mathbf{p}$} \\
\hline & & \multicolumn{2}{|c|}{ Positive } & \multicolumn{2}{|c|}{ Negative } & & \\
\hline & & No. & $\%$ & No. & $\%$ & & \\
\hline \multicolumn{8}{|l|}{ Site of the Tumor: } \\
\hline $\begin{array}{l}\text {-Right side(ascending } \\
\text { caecum) }\end{array}$ & $\begin{array}{l}\text { g colon and } \\
(\mathrm{N}=12)\end{array}$ & 8 & 66.7 & 4 & 33.3 & \multirow{2}{*}{1.118} & \multirow{2}{*}{${ }^{\mathrm{FE}} \mathrm{p}=0.266$} \\
\hline -Left site (descending & $\begin{array}{l}\text { g colon\& rectosigmoid }) \\
(\mathrm{N}=18)\end{array}$ & 15 & 83.3 & 3 & 16.7 & & \\
\hline \multicolumn{8}{|c|}{ Histological type: } \\
\hline -Adenocarcinoma & $(\mathrm{N}=21)$ & 20 & 95.2 & 1 & 4.8 & \multirow{2}{*}{$13.496^{*}$} & \multirow{2}{*}{${ }^{\mathrm{FE}} \mathrm{p}=0.001^{*}$} \\
\hline -Mucinous carcinome & a $\quad(N=9)$ & 3 & 33.3 & 6 & 66.7 & & \\
\hline \multicolumn{8}{|c|}{ Histological grade: } \\
\hline -Low grade & $(\mathrm{N}=17)$ & 16 & 94.1 & 1 & 5.9 & \multirow{2}{*}{0.247} & \multirow{2}{*}{${ }^{\mathrm{FE}} \mathrm{p}=0.810$} \\
\hline -High grade & $(\mathrm{N}=4)$ & 4 & 100.0 & 0 & 0.0 & & \\
\hline \multicolumn{8}{|l|}{ Stage: } \\
\hline Stage I & $(\mathrm{N}=5)$ & 1 & 20 & 4 & 80 & \multirow{3}{*}{$9.352^{*}$} & \multirow{3}{*}{${ }^{\mathrm{MC}} \mathrm{p}=0.007^{*}$} \\
\hline Stage II & $(\mathrm{N}=10)$ & 8 & 80 & 2 & 20 & & \\
\hline Stage III & $(\mathrm{N}=15)$ & 14 & 93.3 & 1 & 6.7 & & \\
\hline
\end{tabular}


Table (4):Correlation between MUC2 expression and clinicopathological parameters in colorectal cancer cases

\begin{tabular}{|c|c|c|c|c|c|c|c|}
\hline \multirow{3}{*}{\multicolumn{2}{|c|}{$\begin{array}{l}\text { Clinicopathological } \\
\text { Variables }\end{array}$}} & \multicolumn{4}{|c|}{ MUC2 Immunoreactivity } & \multirow{3}{*}{$\chi^{2}$} & \multirow{3}{*}{$\mathbf{p}$} \\
\hline & & \multicolumn{2}{|c|}{ Positive } & \multicolumn{2}{|c|}{ Negative } & & \\
\hline & & No. & $\%$ & No. & $\%$ & & \\
\hline \multicolumn{8}{|l|}{ Site of the tumor: } \\
\hline -Right side (ascending & $\begin{array}{l}\text { colon and caecum) } \\
(\mathrm{N}=12)\end{array}$ & 6 & 50.0 & 6 & 50.0 & \multirow{2}{*}{0.833} & \multirow{2}{*}{${ }^{\mathrm{FE}} \mathrm{p}=0.458$} \\
\hline -Left side(descending & $\begin{array}{l}\text { colon \& rectosigmoid) } \\
(\mathrm{N}=18)\end{array}$ & 12 & 66.7 & 6 & 33.3 & & \\
\hline \multicolumn{8}{|l|}{ Histological type: } \\
\hline -Adenocarcinoma & $(\mathrm{N}=21)$ & 9 & 42.9 & 12 & 57.1 & \multirow{2}{*}{$8.571^{*}$} & \multirow{2}{*}{${ }^{\mathrm{FE}} \mathrm{p}=0.004^{*}$} \\
\hline -Mucinous carcinoma & $(\mathrm{N}=9)$ & 9 & 100.0 & 0 & 0.0 & & \\
\hline \multicolumn{8}{|c|}{ Histological grade: } \\
\hline -Low grade & $(\mathrm{N}=17)$ & 7 & 41.2 & 10 & 58.8 & \multirow{2}{*}{0.103} & \multirow{2}{*}{${ }^{\mathrm{FE}} \mathrm{p}=1.000$} \\
\hline -High grade & $(\mathrm{N}=4)$ & 2 & 50.0 & 2 & 50.0 & & \\
\hline \multicolumn{8}{|l|}{ Stage: } \\
\hline Stage I & $(\mathrm{N}=5)$ & 4 & 80.0 & 1 & 20.0 & \multirow{3}{*}{$8.672^{*}$} & \multirow{3}{*}{${ }^{\mathrm{MC}} \mathrm{p}=0.011^{*}$} \\
\hline Stage II & $(\mathrm{N}=10)$ & 9 & 90.0 & 1 & 10.0 & & \\
\hline Stage III & $(\mathrm{N}=15)$ & 5 & 33.3 & 10 & 66.7 & & \\
\hline
\end{tabular}

$\chi 2$ : Chi square test.

MC:Monte Carlo for Chi square test.
FE: Fisher Exact for Chi square test.

*: Statistically significant at $\mathrm{p} \leq 0.05$.

\section{DISCUSSION}

Colorectal cancer is a major public health problem worldwide, although in developed countries survival rates significantly improved over the past two decades, reflecting continuous progress in our understanding of its biology, epidemiology, prevention, early diagnosis and treatment. It arises from interactions among different risk factors that become relevant during the different stages of colorectal carcinogenesis. Various pathological conditions like inflammatory bowel diseases especially ulcerative colitis and adenomatous polyps predispose to CRC development. Novel biomarkers are essential for diagnostic and prognostic tools and to identify patients for targeted therapy ${ }^{(16)}$

The mucus layer coating the gastrointestinal tract is the front line of innate host defense, largely because of the secretory products of intestinal goblet cells. MUC2 is the major secretory mucin synthesized and secreted by goblet cells, whereas goblet and absorptive cells express membrane-bound mucins such as MUC1 in the apical membrane. Deregulation of mucin production in the intestinal tract, contributes to chronic inflammation and tumorigenesis. ${ }^{(17)}$

The aberrant expression of mucins or their alterations in glycosylation are well documented in a variety of inflammatory or malignant human diseases including colorectal cancer and inflammatory bowel disease, making them valuable markers to distinguish between normal and disease conditions. Alterations in mucin expression accompany the development of cancer and influence cellular growth, differentiation, transformation, adhesion, invasion and immune surveillance. Therefore, in an effort to detect and treat cancer at the earliest stage possible, mucins are analyzed as potential markers of disease for diagnosis, progression and are under investigation as therapeutic targets for cancer. ${ }^{(18,19)}$

In the current study, the positive expression rates of MUC1 and MUC2 in normal control were zero and 100\% respectively. In accordance with our findings, Kesari et al, stated that MUC1 was absent in normal colonic mucosa in contrast to MUC2, which was widely expressed by normal mucosa. ${ }^{(20)}$

The present work showed that MUC1 was detected in 6 of 15 cases $(40 \%)$ of UC, whereas the remaining 9 cases $(60 \%)$ did not show MUC1 expression. On the other hand, MUC2 expression was detected in all $15(100 \%)$ cases of UC. In agreement with our results, Sugimoto et al

and Shirazi et al stated that ulcerative colitis exhibits strong expression of MUC2 and weak expression of MUC1 which seem to be similar to normal control. ${ }^{(21,22)}$

Boltin et al reported that MUC2 is the predominant secreted mucin in UC, as in the healthy colon. ${ }^{(23)}$ Shaoul et al suggested that MUC2 expression in goblet cells is preserved in IBD and the loss of goblet cell phenotype is not associated with loss of MUC2 expression. ${ }^{(24)}$ 
In contrast with our findings, Dorofeyev et al observed that the level of expression of MUC2 in all patients with UC was low and correlated with severity of the disease. ${ }^{(25)}$

Sheng et al stated that MUC1 expression was upregulated in severe UC with a reduction in MUC2 expression due to decrease in goblet cell numbers expressing MUC2. ${ }^{(6)}$ This discrepancy in results could be attributed to differences in number of cases, severity of the disease, technique and differences in interpretation between the results of immunohistochemistry, PCR and in situ hybridization.

In the current work, immunohistochemical expression of MUC1 was noted in 5 cases $(33.3 \%)$ of colorectal adenoma, while the remaining 10 cases $(66.7 \%)$ did not show immunoreactivity for MUC1.The five MUC1 positive cases were adenoma with high grade dysplasia, whereas the remaining $10 \mathrm{MUC1}$ negative cases were adenoma with low grade dysplasia. Our results were in line with those observed by Molaei et al who found that MUC1expression was higher with increasing grades of epithelial dysplasia in adenomas. ${ }^{(26)}$ This suggests that MUC1 expression may be an indicator to neoplastic transformation of colorectal mucosa. Other workers reported similar results. ${ }^{(27,28,29)}$

In our series, immunohistochemical expression of MUC2 was observed in 14 of 15 cases $(93.3 \%)$ of colorectal adenomas and only one case $(6.7 \%)$ was negative for MUC2 expression. Previous studies also confirmed similar findings. ${ }^{(30,31,32)}$

In the present study, the positive expression rates of MUC1 and MUC2 in 30 cases of colorectal carcinomas were $76.7 \%$ and $60 \%$ respectively.

In colorectal carcinomas, previous studies showed a wide variation in the expression pattern of MUC1 and MUC2 ranging from $15 \%-77 \%$ for $\mathrm{MUC1}$ and $10 \%-55 \%$ for MUC2. However, all these studies including ours, suggested that there was an overexpression or upregulation of MUC1 expression and a down-regulation of MUC2 expression in CRC. ${ }^{(20,33,34)}$

In the current study, the expression pattern of MUC1 was significantly higher in carcinomas as compared with UC and adenomas $(\mathrm{P} \leq 0.05)$. On the other hand, MUC2 expression was significantly lower in carcinomas, as compared with UC and adenomas $(\mathrm{P} \leq 0.05)$. Thus, our study suggests that up-regulation of MUC1 expression may play a role in the malignant transformation of colorectal tumors, particularly in the late phase of the adenoma-carcinoma sequence from high grade dysplasia to carcinoma, in contrast to MUC2 expression which remained high, except for a significant decrease in carcinoma cases. Other publications also confirmed these findings. ${ }^{(26,29,32)}$

Percinel et al reported that the expression of MUC1 significantly increased in close correlation with the neoplastic process and reached its highest values in cancer group while MUC2 expression showed a significant decrease in cancer group. They suggested that MUC1 plays a role in colorectal carcinogenesis through changes in intracellular signaling, adhesion and migration, and by increasing resistance to apoptosis. MUC2 downregulation observed in carcinomas, however, seems to be related to carcinomatous transformation of the intestinal epithelium, which loses its ability to express the native mucin type due to defective glycosylation observed in the late stages. ${ }^{(27)}$

Sheng et $a \mathrm{l}$ and Boltin et al reported that aberrant mucin expression could play an important role in the pathogenesis of UC-associated neoplasia. They found that MUC1 overexpression and MUC2 suppression influence IBD pathogenesis and progression to cancer colon. ${ }^{(6,23)}$

Regarding histological type of CRC in our series, MUC1 expression was significantly higher in colorectal adenocarcinomas than mucinous carcinomas. MUC2, on the other hand, showed a statistically significant inverse pattern characterized by more expression in mucinous carcinomas than in colorectal adenocarcinomas.

In line with our obtained data, Kim et al and Debunne et al reported that mucinous carcinoma is associated with a higher expression of MUC2, but a lower expression of MUC1. ${ }^{(35,36)}$ In contrast, Abdel-Rahman et al observed that MUC1expression was high in both mucinous and colorectal adenocarcinoma $^{(34)}$ Moreover, $\mathrm{Bu}$ et al stated that decreased MUC2 expression in non-mucinous colon cancer could result from methylation of the MUC2 promoter region with suppression of mucin protein synthesis. ${ }^{(31)}$

As regard histological grade of colorectal adenocarcinoma, the present study showed that there was no significant correlation between the expression of MUC1 or MUC2 and histological grade of colorectal adenocarcinoma. Similar results were obtained by Hadi et al, Khanh et al, and Elzagheid et al. ${ }^{(33,37,38)}$ However, Kesari et al reported that there was no significant difference in MUC2 positivity and tumor grade. On the contrary, MUC1 positivity correlated significantly with tumor grade. ${ }^{(20)}$

The present series demonstrated that there was high significant association between advanced tumor stage and MUC1expression. MUC1 was more frequently expressed in stage III than stage II and stage I. On the other hand, MUC2 showed a statistically significant inverse pattern characterized by more expression in stage I and stage II than stage III.

Previous studies demonstrated that increased MUC1 expression was related to increasing TNM or Dukes stage. ${ }^{33,37)}$ They explained that MUC1 expressed in tumors may function as an anti-adhesion molecule, which inhibits cell to cell adhesion including the release of cells from the tumor.MUC1 expression may be related to 
invasion or metastasis of carcinoma cells in this manner. However, no significant differences in MUC1 or MUC2 expression and stage of CRC has been found in other studies. ${ }^{(20,38)}$ Moreover, Hadi et al and Lugli et al found that loss of MUC2 expression was correlated with increasing stage. ${ }^{(33,39)}$ On the contrary, previous publications reported that positive MUC2 expression was associated with higher stage and development of metastasis. $^{(35,40)}$

This study showed an insignificant association between MUC1or MUC2 expression and the location of the tumor in the large bowel. Similar findings were obtained by previous studies. ${ }^{(20,34)}$ On the contrary, in a study by Li et al, a significant association between high MUC2 expression and tumor location in proximal colon was reported. ${ }^{(41)}$

Many studies reported that overexpression of MUC1 and down-regulation of MUC2 are associated with progression, metastasis and poor prognosis in colorectal adenomas and carcinomas $\left.{ }^{(42,43,44}\right)$ Khanh et al concluded that MUC1 expression appears to be the most useful independent prognostic factor for CRC as compared with the other mucins. ${ }^{(37)}$ However, Kim et al stated that high expression of MUC2 was associated with the metastatic activity of human colon cancer cells. ${ }^{(35)}$

The current study demonstrated that MUC1 expression correlated significantly with advanced tumor stage, thus, MUC1 expression could be indicative of poor outcome in CRC. On the other hand, MUC2 expression correlated significantly with lower TNM stage, hence, MUC2 expression could be related to a favorable outcome in patients with CRC.

Finally, we conclude that up-regulation of MUC1 and down-regulation of MUC2 expression could be involved in carcinogenesis and progression in colorectal tumors and reflect the prognosis to a certain extent. However, further studies of mucin changes in cancer and inflammation are warranted not only as diagnostic and prognostic markers but also as therapeutic targets.

\section{REFERENCES}

1. Parkin DM. Global cancer statistics in the year 2000 . Lancet Oncol 2001;2:533-43.

2. Compton CC.Colorectal carcinoma:diagnostic, prognostic and molecular features. Mod Pathol. 2003;16:376-88.

3. Conteduca V, Sansonno D, Russi S. Precancerous colorectal lesions (Review).Int J Oncol 2013;43(4):973-84.

4. Johansson ME, Ambort D, Schutte A. Composition and functional role of the mucus layers in the intestine. Cell Mol Life Sci 2011;68:3635-41.

5. Bergstrom KS, Xia L. Mucin-type O-glycans and their roles in intestinal homeostasis. Glycobiology 2013;23 (9): 1026-37.

6. Sheng YH, Hasnain SZ, Florin TH. Mucins in inflammatory bowel diseases and colorectal cancer. J Gastroenterol Hepatol 2012;27 (1):28-38.
7. Rachagani S, Torres MP, Batra SK. Current status of mucins in the diagnosis and therapy of cancer. Biofactors 2009;35(6):509-27.

8. Kufe DW. Mucins in cancer:function, prognosis and therapy. Nat Rev Cancer 2009;9 (12):874-85.

9. Johansson ME, Phillipson M. Holm L.The inner of the two MUC2 mucin-dependent mucus layers in colon is devoid of bacteria. Proc Natl Acad Sci USA 2008; 105: $15064-9$.

10. Hollingsworth MA, Swanson BJ. Mucins in cancer: protection and control of the cell surface. Nat Rev Cancer 2004;4(1):45-60.

11. Yonezawa S, Goto M, Yamada N. Expression profiles of MUC1, MUC2 and MUC4 mucins in human neoplasms and their relationship with biological behavior. Proteomics 2008; 16:3329-41.

12. Hamilton Sr, Bosman FT, Boffetta P. Carcinoma of colon and rectum. In:Bosman FT, Hruban RH (eds). WHO classification of tumors of the digestive system. $4^{\text {th }}$ ed. Lyon, France:IARC Press;2010. 134-46.

13. American Joint Committee on Cancer (AJCC). Colon and rectum. In Edge SB, Byrd DR, Compton CC, Fritz AG, Greene FL. AJCC cancer staging manual. $7^{\text {th }}$ ed. New York:Springer;143-64.

14. Kiernan JA. Histological and histochemical methods: theory and practical. $4^{\text {th }}$ ed. Bloxham, UK: Scion;2008.

15. Hsu SM, Rante M, Fauger H.Use of Avidin-biotin peroxidase complex (ABC) in immunoperoxidase techniques: a comparison between $\mathrm{ABC}$ and unlabelled antibody (PAP) procedures. J Histochem Cytochem 1981;29:577-80.

16. Manne U, Shanmugam c, Katkoori VR, Grizzle WE. Development and progression of colorectal neoplasia. Cancer Biomark 2010;9(1-6):235-65.

17. Pelaseyed T, Bergström JH, Gustafsson JK, Ermund A. The mucus and mucins of the goblet cells and enterocytes provide the first defense line of the gastrointestinal tract and interact with the immune system. Immunol Rev 2014;260(1):8-20.

18. Senapati S, Das S, Batra SK. Mucin-interacting proteins: from function to therapeutics. Trends Biochem Sci 2010; 35: 236-45.

19. Carraway KL, Funes M, Workman HC, Sweeney C. Contribution of membrane mucins to tumor progression through modulation of cellular growth signaling pathways. Curr Top Dev Biol 2007;78:1-22.

20. Kesari MV, Gaopande VL, Joshi AR, Gogate BP. Immunohistochemical study of MUC1, MUC2 and MUC5AC in colorectal carcinoma and review of literature. Indian J Gastroenterol 2015;34(1):63-7.

21. Sugimoto K, Ogawa A, Bhan AK, Andoh A.IL-22 ameliorates intestinal inflammation in a mouse model of ulcerative colitis. J Clin Invest 2008;118(2):534-44.

22. Shirazi T, Longman RJ, Corfield AP, Probert CS. Mucins and inflammatory bowel disease. Postgrad Med J 2000;76(898):473-8

23. Boltin D, Perrrets TT, Vilkin A, Niv Y. Mucin function in inflammatory bowel disease: an update. $\mathbf{J}$ Clin Gastroenterol 2013;47(2):106-11.

24. Shaoul R, Okada Y, Cutz E, Marcon MA. Colonic expression of MUC2, MUC5AC, and TFF1 in inflammatory bowel disease in children. $\mathrm{J}$ Pediatr Gastroenterol Nutr 2004;38(5):488-93. 
25. Dorofeyev AE, Vasilenko IV, Rassokhina OA, Kondratiuk RB. Mucosal barrier in ulcerative colitis and Crohn's disease. Gastroenterol Res Pract 2013; 2013: 431231.

26. Molaei M, Mansoori BK, Vahedi M, Fatemi SR. Mucins in neoplastic spectrum of colorectal polyps:can they provide predictions? BMC Cancer 2010; 10:537.

27. Percinel S, Savas B, Ensari A, Bektas M. Mucins in the colorectal neoplastic spectrum with reference to conventional and serrated adenomas. Turk J Gastroenterol 2007;18 (4):230-8.

28. Silk AW, Schoen RE, Potter D, Finn OJ. Humoral immune response to abnormal MUC1 in subjects with colorectal adenoma and cancer. Mol Immunol 2009;47 (1):52-6.

29. Kimura T, Mc Kolanis, Islam K, Salazar AM. MUC1 vaccine for individuals with advanced adenoma of the colon: a cancer immunoprevention feasibility study. Cancer Prev Res 2013;6 (1):18-26.

30. Mizoshita T, Inada KI, Hirano N, Tajika M. Loss of MUC2 expression correlates with progression along the adenoma-carcinoma sequence pathway as well as de novo carcinogenesis in the colon. Histol Histopathol 2007;22 (3):251-60.

31. $\mathrm{Bu} \mathrm{XD,} \mathrm{Li} \mathrm{N,Tian} \mathrm{XQ,} \mathrm{Li} \mathrm{L.Altered} \mathrm{expression} \mathrm{of}$ MUC2 and MUC5AC in progression of colorectal carcinoma.World J Gastroenterol 2010; 16(32):4089-94.

32. El-Sayed IH, Lotfy M, Moawad M, Immunodiagnostic potential of mucin ( MUC2) and Thomsen-Friedenreich (TF) antigens in Egyptian patients with colorectal cancer. Eur Rev Med Pharmacol Sci 2011 J;15 (1):91-7.

33. Hadi NI, Shakoor KA, Kafil N. Immunohistochemical study of mucins in colorectal carcinoma: could it be a guide for biological behavior? J Surgery Pakistan 2009; 14(2):103-7.

34. Abdel-Rahman A, Khodeir M, Belal Saied, Osman H. Immunohistochemical Expression of Cytokeratins 7 and 20 as well as Mucins 1 and 2 in Egyptian cases of primary colorectal carcinomas. World Applied Sciences Journal 2010;8(1):10-20.
35. Kim YS, Ho SB. Intestinal goblet cells and mucins in health and disease: recent insights and progress. Curr Gastroenterol Rep 2010;12 (5):319-30.

36. Debunne H, Ceelen W. Mucinous differentiation in colorectal cancer: molecular, histological and clinical aspects. Acta Chir Belg $2013 ; 113(6): 385-90$

37. Khanh do T, Meeekata E, Shimizu T, Shiomi H. Transmembrane mucin MUC1 overexpression and its association with CD10 myeloid cells transforming growth factor- $\beta 1$ expression and tumor budding grade in colorectal cancer. Cancer Sci $2013 ; 104(7): 958-64$.

38. Elzagheid A, Emaetig F, Buhmeida A, Laato M. Loss of MUC2 expression predicts disease recurrence and poor outcome in colorectal carcinoma. Tumour Biol 2013; 34 (2): $621-8$

39. Lugli A, Zlobec I, Baker K, Minoo P. Prognostic significance of mucins in colorectal cancer with different DNA mismatch-repair status. J Clin Pathol 2007; 60 (5): 534-9.

40. Walsh MD, Pearson SA, Walters RJ, Nagler B. Expression of MUC2, MUC5AC, MUC5B, and MUC6 mucins in colorectal cancers and their association with the $\mathrm{CpG}$ island methylator phenotype. Mod Pathol 2013; 26 (12):1642-56.

41. Li L, Huang PL, Yu XJ, BU XD. Clinicopathological significance of mucin 2 immuno-histochemical expression in colorectal cancer: a meta-analysis. Chin J Cancer Res 2012;24(3):190-5

42. Nath S, Mukherjee P. MUC1: a multifaceted oncoprotein with a key role in cancer progression. Trends Mol Med 2014; 20 (6):332-42.

43. Kang H, Min BS, Lee KY, Kim SN. Loss of E-cadherin and MUC2 expressions correlated with poor survival in patients with stages II and III colorectal carcinoma. Ann Surg Oncol 2011;18(3):711-9.

44. Jonckheere N., Van Seuningen I. The membrane-bound mucins: From cell signaling to transcriptional regulation and expression in epithelial cancers. Biochimie 2010;92 (1):1-11. 